\title{
Dexmedetomidine alleviates inflammation in neuropathic pain by suppressing NLRP3 via Nrf2 activation
}

\author{
WENYAN SHAN ${ }^{1,2}$, XIAOYUN LIAO ${ }^{1,2}$, YIXUN TANG ${ }^{1,2}$ and JITONG LIU ${ }^{1,2}$ \\ ${ }^{1}$ Department of Anesthesiology, Hunan Provincial People's Hospital (The First Affiliated Hospital of \\ Hunan Normal University); ${ }^{2}$ Clinical Research Center for Anesthesiology of ERAS \\ in Hunan Province, Changsha, Hunan 410005, P.R. China
}

Received October 13, 2020; Accepted June 24, 2021

DOI: $10.3892 / \mathrm{etm} .2021 .10479$

\begin{abstract}
The aim of the present study was to investigate the mechanism by which dexmedetomidine (DEX) alleviates neuropathic pain in a chronic constriction injury (CCI) model in rats. A CCI rat model was established through sciatic nerve ligation. CCI rats were treated with DEX, the nuclear factor erythroid 2-related factor 2 (Nrf2) inhibitor ML385, the NLR family pyrin domain containing 3 (NLRP3) antagonist MCC950 and/or the NLRP3 activator nigericin. The mechanical withdrawal threshold (MWT) was measured to assess the pain sensitivity of CCI rats. Hematoxylin and eosin staining and TUNEL staining were used to examine spinal injury and apoptosis, respectively. ELISA was used to quantify the levels of inflammatory factors. The expression levels of Nrf2 and NLRP3 were also examined. The results indicated that a decrease in MWT and increases in spinal cord injury, apoptosis and inflammatory factors were detected in CCI rats compared with control rats. Spinal inflammation was abrogated in DEX-treated CCI rats. Compared with the model group, an increase in MWT and decreases in spinal cord injury, apoptosis and inflammatory factors were detected in rats treated with MCC950, while the opposite effects were observed in rats treated with nigericin. The opposite effects on these indicators were observed in the DEX + ML385 and MCC950 + ML385 groups compared with the DEX and MCC950 groups, respectively. MWT was increased, while spinal cord injury, apoptosis and inflammation decreased in the nigericin + DEX group compared with the nigericin group. In summary, the results of the present study indicated that
\end{abstract}

Correspondence to: Dr Jitong Liu, Department of Anesthesiology, Hunan Provincial People's Hospital (The First Affiliated Hospital of Hunan Normal University), 61 West Jiefang Road, Furong, Changsha, Hunan 410005, P.R. China

E-mail: liujitong1971@126.com

Key words: neuropathic pain, dexmedetomidine, chronic constriction injury, nuclear factor erythroid 2-related factor 2, NLR family pyrin domain containing 3
DEX reduced neuropathic pain in CCI rats by suppressing NLRP3 through Nrf2 activation.

\section{Introduction}

Neuropathic pain is caused by a lesion or disease of the somatosensory system (1) and characterized by positive and negative sensory symptoms, such as the gain and loss of somatosensory function, which occurs alone or in different combinations (2). Neuropathic pain results from etiologically diverse disorders affecting the peripheral or central nervous system or neurological conditions of unknown etiology, and it either persists continuously or presents as recurrent painful episodes (3). Chronic neuropathic pain may complicate neuropathic symptoms and treatment decisions, leading to poor outcomes and impaired patient quality of life (4). The diagnosis of neuropathic pain is also complex due to diverse clinical features that are categorized based on anatomical (such as peripheral and central) and etiological (such as degenerative, traumatic, infectious metabolic and toxic) factors (5). Further research and clinical trials should be implemented to improve the diagnosis and treatment of neuropathic pain. The present study aimed to validate the anti-inflammatory and analgesic effects of dexmedetomidine (DEX) on neuropathic pain in a rat model of chronic constriction injury (CCI).

DEX is a potent and selective $\alpha 2$-adrenoceptor agonist with sedative, anxiolytic, sympatholytic and analgesic effects (6). This drug is used to induce short- and long-term sedation in patients in intensive care units and can effectively suppress delirium (7). DEX has been reported to have the potential to prevent acute pain in adults who undergo abdominal surgery (8). Moreover, a steadily increasing number of studies have investigated the inhibitory effect of DEX on neuropathic pain. For example, DEX suppressed neuropathic pain by inhibiting $\mathrm{P} 2 \mathrm{X}$ purinoceptor 7 through ERK regulation in a rat model of CCI (9). DEX can protect the nervous system via anti-inflammatory, anti-excitotoxic, antiapoptotic, antioxidative and other mechanisms (10).

NLR family pyrin domain containing 3 (NLRP3), which belongs to the nucleotide-binding oligomerization domain-like receptor family of proteins, is a pattern recognition receptor that contributes to the formation of inflammasomes (11). Among all inflammasomes, the NLRP3 inflammasome 
has been extensively investigated and verified to serve a critical role in innate immunity and the pathology of human diseases (12). NLRP3 promotes inflammation through the cleavage of the proinflammatory cytokines IL-1 $\beta$ and IL-18 by stimulating caspase-1 (13). Grace et al (14) revealed that morphine promoted the intensity and duration of neuropathic pain by activating the NLRP3 inflammasome. DEX has been indicated to modulate the NLRP3 inflammasome in animal models of acute lung and kidney injury $(15,16)$. However, whether NLRP3 is implicated in the DEX-induced reduction in inflammatory responses and neuropathic pain has not been yet fully elucidated.

Nuclear factor-erythroid 2-related factor 2 (Nrf2) is a multifunctional protein that modulates antioxidant and other cytoprotective genes (such as GPX2 and GCLC) and is involved in inflammatory processes $(17,18)$. Plumbagin, a naphthoquinone derived from the plant Plumbago, has been indicated to alleviate CCI-induced neuropathic pain by upregulating Nrf2 (19). In addition, the new biphenyl diester derivative $\mathrm{AB}-38 \mathrm{~b}$ has been demonstrated to attenuate diabetic nephropathy by suppressing NLRP3 via Nrf2 activation (20). However, the Nrf2 signaling-mediated regulation of NLRP3 in neuropathic pain has rarely been investigated. A previous study revealed that DEX exerted neuroprotective effects via the Nrf2 signaling pathway (21). The present study aimed to investigate whether DEX-induced activation of Nrf2 further regulated NLRP3 to ameliorate neuropathic pain.

\section{Materials and methods}

Animal experiments. Male Sprague-Dawley (SD) rats $(\mathrm{n}=138$; weight, 200-220 g; age, 2 months) were purchased from Hunan SJA Laboratory Animal Co., Ltd. The rats were subjected to a $12 / 12 \mathrm{~h}$ light/dark cycle at room temperature $\left(23 \pm 1^{\circ} \mathrm{C}\right)$ and $60-65 \%$ humidity and were provided standard food and water for at least 1 week. All animal experiments were approved by the Animal Protection and Use Committee of Hunan Provincial People's Hospital (Changsha, China) and strictly followed the 'Guidelines for the Use and Management of Laboratory Animals' issued by the National Institutes of Health and Animal Research: Reporting In Vivo Experiments Guidelines (22). Adult male and female rats respond differentially to harmful stimuli. Among rodents, females are more sensitive compared with males to noxious stimuli, and males generally exhibit stronger stress-induced analgesia (23). Therefore, male rats were used for the experiments in the present study.

A total of 138 SD rats were divided into different groups in a randomized manner ( 6 rats per group). Rats in control groups $(n=6 \times 2)$ were left untreated. Rats in sham groups $(n=6 \times 3)$ underwent surgery, during which no nerve ligation was performed. Rats in the model group were subjected to sciatic nerve ligation for induction of CCI after being anesthetized with pentobarbital sodium (40 mg/kg). The exposed sciatic nerves were separated and ligated with four 4-0 chromic gut suture (Ethicon, Inc.).

After the model establishment, the rats in each group were subjected to the corresponding treatment. The groups were as follows: Model group ( $n=6 \times 3$; untreated model rats), $\mathrm{DEX}+1$ group $(\mathrm{n}=6$; intraperitoneally injected with $1 \mu \mathrm{g} / \mathrm{kg}$
DEX every day for 7 days after the operation; Jiangsu Nhwa Pharmaceutical Co., Ltd.), DEX+2 ( $n=6$; intraperitoneally injected with $2 \mu \mathrm{g} / \mathrm{kg}$ DEX for 7 days post-operation), DEX+5 group ( $\mathrm{n}=6$; intraperitoneally injected with $5 \mu \mathrm{g} / \mathrm{kg} \mathrm{DEX}$ for 7 days post-operation), DEX group ( $n=6 \times 2$; intraperitoneally injected with $5 \mu \mathrm{g} / \mathrm{kg}$ DEX for 7 days post-operation), MCC950 group [n=6x2; intraperitoneally injected with $50 \mu \mathrm{g} / \mathrm{kg}$ MCC950 (NLRP3 antagonist) for 7 days post-operation; MedChemExpress], nigericin group [n=6x2; intraperitoneally injected with $1 \mathrm{mg} / \mathrm{kg}$ nigericin (NLRP3 activator) for 7 days post-operation; MedChemExpress], DEX + ML385 group [n=6x2; intraperitoneally injected with $5 \mu \mathrm{g} / \mathrm{kg}$ DEX and $30 \mathrm{mg} / \mathrm{kg}$ ML385 (Nrf2 inhibitor) for 7 days post-operation; Selleck Chemicals], DEX + nigericin group ( $\mathrm{n}=6 \mathrm{x} 2$; intraperitoneally injected with $5 \mu \mathrm{g} / \mathrm{kg} \mathrm{DEX}$ and $1 \mathrm{mg} / \mathrm{kg}$ nigericin for 7 days post-operation) and MCC $950+$ ML385 group ( $\mathrm{n}=6 \times 2$; intraperitoneally injected with $30 \mathrm{mg} / \mathrm{kg}$ ML385 and $50 \mu \mathrm{g} / \mathrm{kg}$ MCC950 for 7 days post-operation). The rats were deeply anesthetized with an intraperitoneal injection of $50 \mathrm{mg} / \mathrm{kg}$ thiopental sodium and euthanatized via cervical dislocation. Subsequently, $\sim 1 \mathrm{ml}$ of blood was collected from each rat and centrifuged at $1,000 \mathrm{x} \mathrm{g}, 4^{\circ} \mathrm{C}$ for $10 \mathrm{~min}$. The blood samples were stored at $-20^{\circ} \mathrm{C}$. The L4-L6 spinal cords were collected from the rats and immediately stored in an ultra-low temperature refrigerator $\left(-80^{\circ} \mathrm{C}\right)$. The experimental timetable is presented in Fig. 1.

Animal behavioral analysis. The rats were subjected to behavioral tests on day $0,1,3,7$ and 14 post-operatively. These rats were randomly assigned to the control, sham, model, DEX +1 , $\mathrm{DEX}+2, \mathrm{DEX}+5, \mathrm{DEX}, \mathrm{MCC} 950$, nigericin, DEX + ML385, MCC950 + ML385 and DEX + nigericin groups and were treated as aforementioned ( $n=6$ per group). The behavioral tests were conducted between 9:00 and 12:00 a.m. in a quiet environment. The rats were placed in wire cages for $30 \mathrm{~min}$ before the experiment. The mechanical withdrawal threshold (MWT) of the rats was measured three times at an interval of 5 min using an automated dynamic plantar aesthesiometer (Ugo Basile S.R.L.). The minimum retraction force $(\mathrm{G})$ of the rats' right hind paw was recorded, and the average value of the power that induced a reliable retreat was recorded as the threshold. Quick withdrawal or licking of the paws in response to stimuli was considered a positive reaction.

Hematoxylin and eosin $(H \& E)$ staining. L4-L6 spinal cords were collected from rats in the control, sham, model, DEX, MCC950, nigericin, DEX + ML385, MCC950 + ML385 and $\mathrm{DEX}+$ nigericin groups ( $\mathrm{n}=6$ per group) 7 days post-operation. The spinal cords were fixed in $4 \%$ paraformaldehyde at $4^{\circ} \mathrm{C}$ for $48 \mathrm{~h}$ and then dehydrated in $30 \%$ sucrose at $4^{\circ} \mathrm{C}$ overnight. The spinal cords were embedded in paraffin and cut into $5-\mu \mathrm{m}$ sections for H\&E staining. At room temperature, the sections were sequentially immersed in xylene for $5 \mathrm{~min}, 100 \%$ ethanol for $10 \mathrm{~min}, 95 \%$ ethanol for $5 \mathrm{~min}$ and $75 \%$ ethanol for $5 \mathrm{~min}$ and finally washed with distilled water. The deparaffinized sections were stained with hematoxylin at room temperature for $5 \mathrm{~min}$, soaked in deionized water for $5 \mathrm{~min}$, then stained with eosin for $1 \mathrm{~min}$. Finally, the spinal cord sections were observed using a light microscope (ABX50; Olympus Corporation). The scoring criteria of spinal cord injury were as follows: $0=\mathrm{No}$ 


\begin{tabular}{|c|c|c|c|c|}
\hline 0 day & 1 day & 3 days & 7 days & 14 days \\
\hline $\begin{array}{l}138 \text { rats } \\
\text { underwent } \\
\text { surgery and } \\
\text { were treated } \\
\text { with drugs; } \\
84 \text { rats } \\
\text { underwent } \\
\text { behavioral } \\
\text { test }\end{array}$ & $\begin{array}{l}138 \text { rats } \\
\text { were } \\
\text { treated } \\
\text { with drugs; } \\
84 \text { rats } \\
\text { underwent } \\
\text { behavioral } \\
\text { test }\end{array}$ & $\begin{array}{l}138 \text { rats } \\
\text { were } \\
\text { treated } \\
\text { with drugs; } \\
84 \text { rats } \\
\text { underwent } \\
\text { behavioral } \\
\text { test }\end{array}$ & $\begin{array}{l}138 \text { rats were treated } \\
\text { with drugs; } 84 \text { rats } \\
\text { underwent behavioral } \\
\text { test; collection of } \\
\text { blood samples from } \\
\text { another } 54 \text { rats and } \\
\text { sacrifice of these } 54 \\
\text { rats and isolation of } \\
\text { the L4-L6 part of } \\
\text { spinal cord to perform } \\
\text { other experiments }\end{array}$ & $\begin{array}{l}84 \text { rats were treated } \\
\text { with drugs and } \\
\text { underwent behavioral } \\
\text { test }\end{array}$ \\
\hline
\end{tabular}

Figure 1. Experimental schedule. A total of 138 rats were included in the present study. A total of 84 rats underwent behavioral tests on day $0,1,3,7$ and 14 after the operation, including the DEX+1, DEX+2 and DEX+5 groups, two sham groups, two model groups and one control, DEX, MCC950, nigericin, DEX + ML385, MCC950 + ML385 and DEX + nigericin groups ( $\mathrm{n}=6$ rats per group). A total of 54 rats were sacrificed for spinal cord collection 7 days after the drug administration, including one control, sham, model, DEX, MCC950, nigericin, DEX + ML385, MCC950 + ML385 and DEX + nigericin groups (n=6 rats per group).

lesion; 1=gray matter containing 1-5 eosinophilic neurons; $2=$ gray matter containing 6-10 eosinophilic neurons; $3=$ gray matter containing $>10$ eosinophilic neurons; $4=$ infarction of $<1 / 3$ of the gray matter area; $5=$ infarction of $1 / 3-1 / 2$ of the gray matter area; and $6=$ infarction of $>1 / 2$ of the gray matter area.

TUNEL staining. L4-L6 spinal cords were collected from rats in the control, sham, model, DEX, MCC950, nigericin, DEX + ML385, MCC950 + ML385 and DEX + nigericin groups ( $\mathrm{n}=6$ per group) 7 days post-operation. The tissues were fixed, dehydrated, paraffin embedded and sliced as aforementioned. Paraffin sections of the spinal cords were dewaxed with xylene and hydrated using alcohol gradients (100\% ethanol, $10 \mathrm{~min}$; 95\%, $5 \mathrm{~min}$; 75\%, $5 \mathrm{~min}$; distilled water). Subsequently, the spinal cord sections were treated with proteinase $\mathrm{K}$ at room temperature for $30 \mathrm{~min}$ and immersed in enhanced endogenous peroxidase blocking solution (100\%; cat. no. P0100B; Beyotime Institute of Biotechnology) at room temperature for $10 \mathrm{~min}$. The tissues were incubated with TUNEL detection solution (cat. no. C1091; Beyotime Institute of Biotechnology) for $60 \mathrm{~min}$ at $37^{\circ} \mathrm{C}$ in the dark. Finally, the spinal cord sections were washed with PBS three times, mounted with neutral balsam and observed using a light microscope. A total of three non-overlapping fields were randomly selected from each slice. The apoptosis rate (\%) was determined as the number of brown cells/total number of cells $\times 100 \%$.

Reverse transcription-quantitative ( $R T-q) P C R$. Total RNA was extracted from the L4-L6 spinal cords of rats in the control, sham, model, DEX and DEX + ML385 groups ( $\mathrm{n}=6$ per group) 7 days post-operation using TRIzol $^{\circledR}$ reagent (Invitrogen; Thermo Fisher Scientific, Inc.) according to the manufacturer's instructions. RNA was reverse transcribed into cDNA using a Universal cDNA Synthesis kit (Promega Corporation) according to the manufacturer's instructions. Gene expression was analyzed using a LightCycler 480 (Roche Diagnostics) in accordance with the instructions of the fluorescence quantitative PCR kit (SYBR Green PCR kit; Takara Bio, Inc.). The reaction conditions were as follows: 5 min of pre-denaturation at $95^{\circ} \mathrm{C}$, followed by 40 cycles of $10 \mathrm{sec}$ of denaturation at $95^{\circ} \mathrm{C}$, $10 \mathrm{sec}$ of annealing at $60^{\circ} \mathrm{C}$ and $20 \mathrm{sec}$ of extension at $72^{\circ} \mathrm{C}$. Relative quantification was performed using the comparative $2^{-\Delta \Delta C q}$ method (24), with GAPDH as an internal reference. The primer sequences used are presented in Table I.

Western blotting. The L4-L6 segments of the spinal cords were collected from rats in the control, sham, model, DEX, MCC950, nigericin, DEX + ML385, MCC950 + ML385 and $\mathrm{DEX}+$ nigericin groups ( $\mathrm{n}=6$ per group) 7 days post-operation and mixed with RIPA buffer (Beyotime Institute of Biotechnology) containing protease and phosphatase inhibitors. Total protein was extracted from the lysates after centrifugation at $8,759 \mathrm{xg}$ for $30 \mathrm{~min}$ at $4^{\circ} \mathrm{C}$, or nucleoproteins were extracted using a nuclear protein extraction kit (cat. no. R0050, Beijing Solarbio Science \& Technology Co., Ltd.) according to the manufacturer's instructions. Protein concentration was measured using a BCA kit. The proteins $(100 \mu \mathrm{g} /$ well $)$ were separated using $8 \%$ SDS-PAGE and transferred onto a PVDF membrane. The membrane was immersed in 5\% non-fat milk at room temperature for $1 \mathrm{~h}$ and then incubated with primary antibodies against GAPDH (1:10,000; cat. no. ab181602), Nrf2 (1:100; cat. no. ab137550) and NLRP3 (1:500; cat. no. ab214185) (all Abcam) at $4^{\circ} \mathrm{C}$ overnight. After being washed with $0.1 \%$ PBS-Tween-20 (TBST) three times, the membrane was incubated with horseradish peroxidase-labeled goat anti-rabbit IgG secondary antibody (1:5,000; cat. no. CW0103; CoWin Biosciences) at room temperature for $30 \mathrm{~min}$. Finally, the membrane was washed with TBST four times and subjected to color development with ECL (Proanti Biotechnology Development Co., Ltd.). The blots were detected by a chemiluminescence imaging system (GE Healthcare). The greyscale of blots was analyzed by ImageJ (version 1.46; National Institutes of Health).

ELISA. Whole blood of rats in the control, sham, model, DEX, MCC950, nigericin, DEX + ML385, MCC950 + ML385 and 
Table I. Primer sequences.

\begin{tabular}{lc}
\hline Primer name & \multicolumn{1}{c}{ Sequence, 5'-3' } \\
\hline Nrf2 forward & AGGTTGCCCACATTCCCAAA \\
Nrf2 reverse & AGTGACTGAAACGTAGCCGA \\
GAPDH forward & TTCTGGGATACACGGAGCAC \\
GAPDH reverse & TACCAGCACCAGCGTCAAAG
\end{tabular}

Nrf2, nuclear factor erythroid 2-related factor 2.

$\mathrm{DEX}+$ nigericin groups ( $\mathrm{n}=6$ per group) was mixed with EDTA and then centrifuged at $1,000 \mathrm{x}$ g for $10 \mathrm{~min}$ at $4^{\circ} \mathrm{C}$ to collect the supernatant. The expression levels of TNF- $\alpha$ (cat. no. RTA00), IL-1 $\beta$ (cat. no. RLB00), IL-6 (cat. no. R6000B) and IL-10 (cat. no. R1000) were measured using commercial ELISA kits (R\&D Systems, Inc.) in accordance with the manufacturer's instructions.

Statistical analysis. Statistical analysis was performed using GraphPadPrism 7.0(GraphPadSoftware,Inc.).Histopathological scores are presented as the median \pm interquartile range and were analyzed using Kruskal-Wallis followed by Dunn's post hoc test. All other data were normally distributed and are presented as the mean \pm standard deviation. Unpaired Student's t-test was used to compare differences between two groups. One-way ANOVA followed by Tukey's post hoc test was used for multigroup comparisons. $\mathrm{P}<0.05$ was considered to indicate a statistically significant difference.

\section{Results}

DEX reduces neuropathic pain in CCI rats. CCI rats were injected with different concentrations of DEX $(1,2$ and $5 \mu \mathrm{g} / \mathrm{kg})$ to determine the optimal dose for subsequent experiments. MWT was measured to assess the pain sensitivity of the rats. Compared with the sham group, the model group exhibited a significant decrease in the MWT (Fig. 2A; $\mathrm{P}<0.01$ ). Compared with the model group, the DEX+1 group demonstrated no significant change in the MWT, while the DEX+2 group and DEX+5 group exhibited significantly increased MWTs (Fig. 2A; both $\mathrm{P}<0.05$ ). Among the three groups, the $\mathrm{DEX}+5$ group demonstrated the highest increase in the MWT. Therefore, $5 \mu \mathrm{g} / \mathrm{kg}$ DEX was used for subsequent experiments.

CCI rats were injected with $5 \mu \mathrm{g} / \mathrm{kg}$ DEX. As demonstrated in Fig. 2A, the MWT was decreased in the model group compared with the control group $(\mathrm{P}<0.01)$, but increased in the DEX group compared with the model group $(\mathrm{P}<0.05)$. This result indicated that the rats in the model group had the highest pain sensitivity, whereas DEX reduced the pain sensitivity of CCI rats.

H\&E staining demonstrated that spinal injury was increased in the model group compared with the control group $(\mathrm{P}<0.001)$ but was reduced in the DEX group compared with the model group ( $\mathrm{P}<0.01$; Fig. 2B). The number of TUNEL-positive cells in the spinal cord was increased in the model group compared with the control group $(\mathrm{P}<0.001)$ but was decreased in the DEX group compared with the model group $(\mathrm{P}<0.01)$ (Fig. $2 \mathrm{C}$ ).
TNF- $\alpha$, IL-1 $\beta$ and IL-6 expression levels were increased in the model group compared with the control group $(\mathrm{P}<0.01)$, but were decreased in the DEX group compared with the model group $(\mathrm{P}<0.05)$ (Fig. 2D). The expression patterns of IL-10 were the opposite of those of TNF- $\alpha$, IL-1 $\beta$ and IL- 6 in the model and DEX groups (Fig. 2D; $\mathrm{P}<0.01$ vs. the control group and $\mathrm{P}<0.05$ vs. the model group). RT-qPCR and western blotting results demonstrated that the expression levels of Nrf 2 mRNA and protein were downregulated in the model group compared with the control group (Fig. 2E, $\mathrm{P}<0.001$; Fig. $2 \mathrm{~F}, \mathrm{P}<0.01$ ), but were upregulated in the DEX group compared with the model group (Fig. 2E, P<0.01; Fig. 2F, P<0.05). Moreover, the protein expression level of NLRP3 was upregulated in the model group compared with the control group, but was downregulated in the DEX group compared with the model group (Fig. 2F; both $\mathrm{P}<0.01)$. There was no significant difference between the sham group and the control group. These results demonstrated that DEX suppressed inflammation and apoptosis in the spinal cord to reduce neuropathic pain in CCI rats.

NLRP3 increases neuropathic pain in CCI rats. CCI rats were injected with the NLRP3 antagonist MCC950 or the NLRP3 activator nigericin. Western blotting revealed downregulation of NLRP3 in the MCC950 group and upregulation of NLRP3 in the nigericin group compared with the model group (Fig. 3A; both $\mathrm{P}<0.01$ ). These results demonstrated the successful suppression or activation of NLRP3 in CCI rats. MWT was significantly increased in the MCC950 group (Fig. 3B; $\mathrm{P}<0.01$ ), but was significantly decreased in the nigericin group compared with the model group (Fig. 3B; $\mathrm{P}<0.05$ ). $H \& E$ staining indicated that spinal injury was reduced in the MCC950 group but was increased in the nigericin group compared with the model group (Fig. $3 \mathrm{C}$; both $\mathrm{P}<0.01$ ). The TUNEL staining results indicated that, compared with the model group, the apoptosis rate was significantly decreased in the MCC950 group, but was increased in the nigericin group (Fig. 3D; both $\mathrm{P}<0.01$ ). Decreases in the expression levels of TNF- $\alpha$, IL-1 $\beta$ and IL- 6 and an increase in IL-10 were observed in the MCC950 group compared with the model group, while the inverse expression patterns of these cytokines were detected in the nigericin group (Fig. 3E; P<0.05). These results suggested that NLRP3 could promote neuropathic pain in CCI rats.

DEX reduces neuropathic pain by activating Nrf2 in CCI rats. CCI rats were injected with the Nrf2 inhibitor ML385 together with DEX. RT-qPCR and western blotting demonstrated that Nrf2 was downregulated in the DEX + ML385 group compared with the DEX group, indicating the effectiveness of ML385 (Fig. 4A, P<0.001; Fig. 4B, P<0.01). The DEX + ML385 group exhibited significantly decreased MWT compared with the DEX group (Fig. 4C; $\mathrm{P}<0.05$ ). H\&E and TUNEL staining demonstrated that spinal injury and apoptosis rate were exacerbated in the DEX + ML385 group compared with the DEX group (Fig. 4D, $\mathrm{P}<0.05$; Fig. 4E, $\mathrm{P}<0.01$ ). The expression levels of TNF- $\alpha$, IL- $1 \beta$ and IL- 6 were increased, while the expression of IL-10 was decreased in the DEX + ML385 group compared with the DEX group (Fig. 4F; all $\mathrm{P}<0.05$ ). Moreover, the expression level of NLRP3 was also upregulated in the DEX + ML385 group compared with the DEX group (Fig. 4G; 

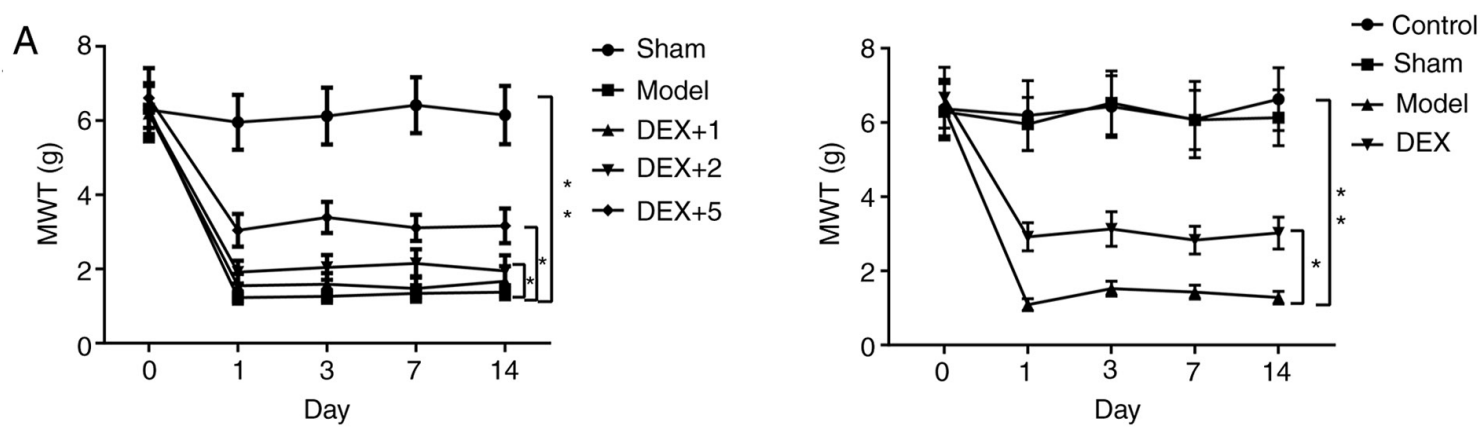

B
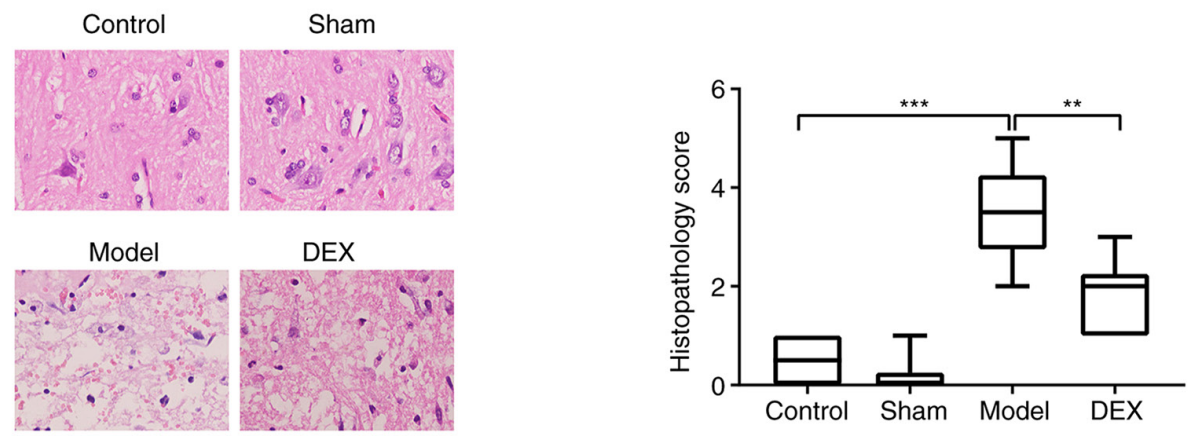

C
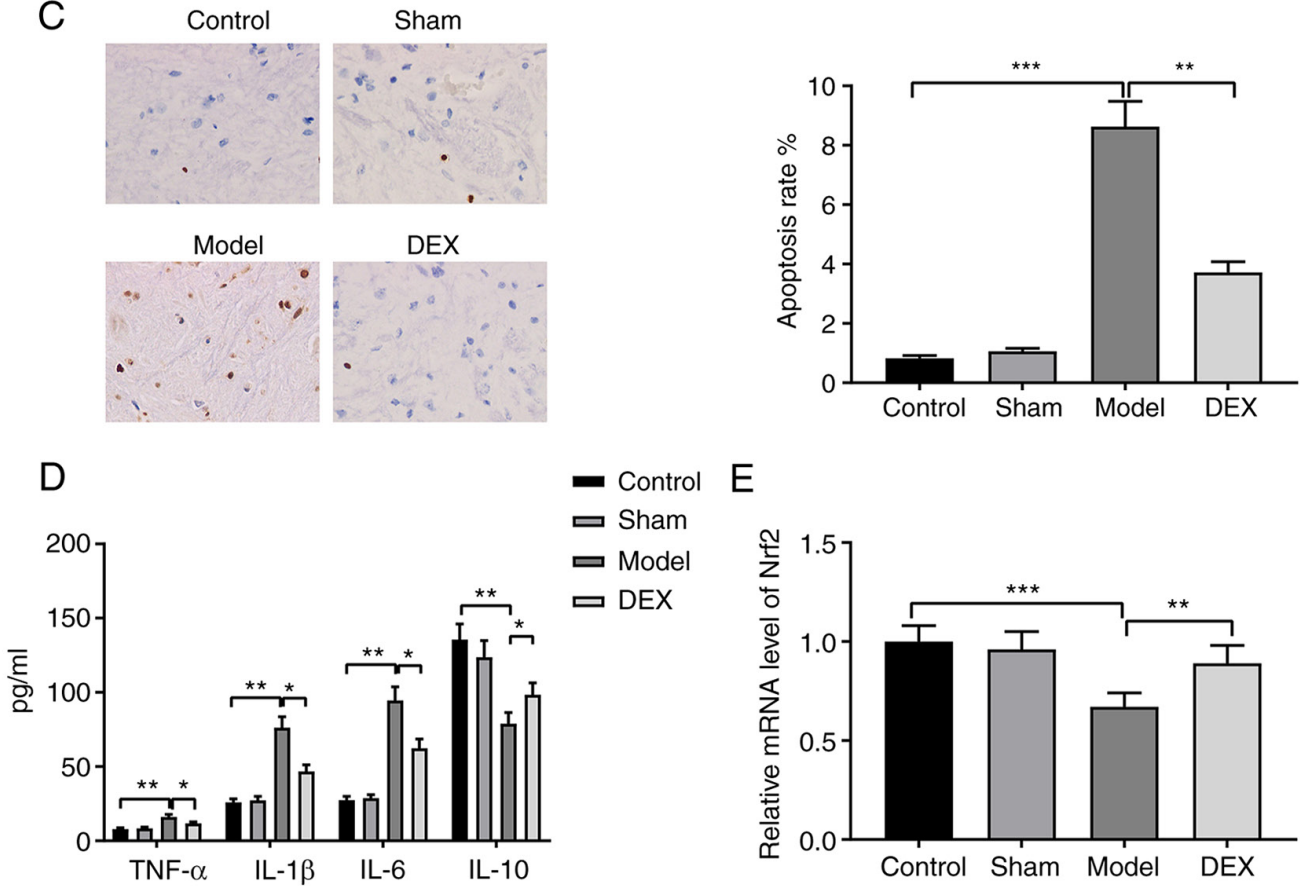

Control E ص Sham 口 Model ص DEX

$\mathrm{F}$

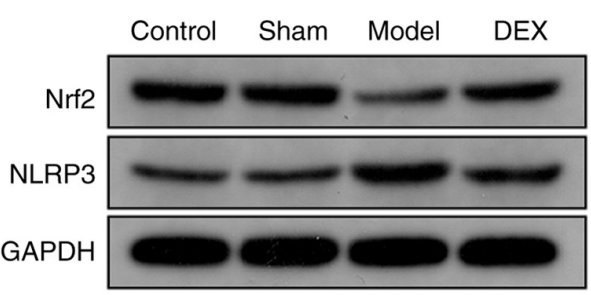

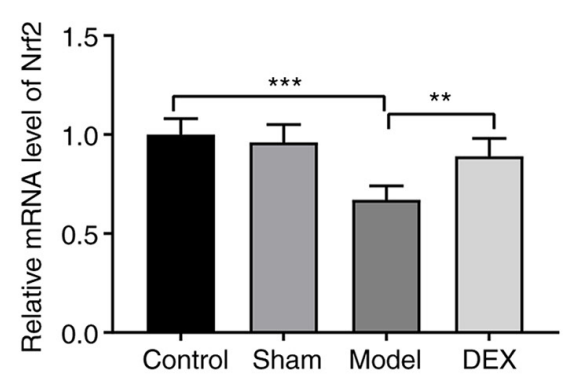

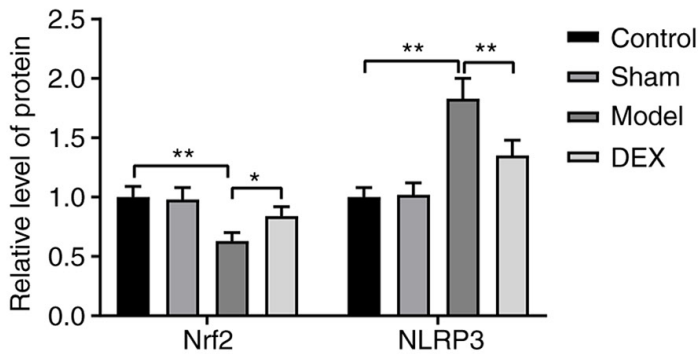

Figure 2. DEX reduces neuropathic pain in CCI rats. (A) CCI rats were injected with 1,2 or $5 \mu \mathrm{g} / \mathrm{kg}$ DEX before MWT of the rats was measured. (B) CCI rats were injected with $5 \mu \mathrm{g} / \mathrm{kg}$ DEX and hematoxylin and eosin staining revealed the spinal cord injury (magnification, $\mathrm{x} 400$ ). Each set of data was arranged from $\mathrm{X} 1$ to $\mathrm{Xn}$ in ascending order. The median values were equal $(\mathrm{Xn} / 2+\mathrm{Xn} / 2+1) / 2$ when ' $\mathrm{n}$ ' was even. The number of rats in each group was even ( $\mathrm{n}=6)$, so the median values were the average of X3+X4. (C) TUNEL staining was performed to examine the apoptosis in the spinal cords (magnification, $x 400$ ). (D) The expression levels of inflammatory factors were measured via ELISA. (E) Reverse transcription-quantitative PCR was performed to detect the expression of Nrf2. (F) Western blotting was performed to detect the expression levels of Nrf2 and NLRP3. $n=6$ per group. $P<0.05 ;{ }^{* *} \mathrm{P}<0.01 ;{ }^{* * * *} \mathrm{P}<0.001$. DEX, dexmedetomidine; CCI, chronic constriction injury; MWT, mechanical withdrawal threshold; Nrf2, nuclear factor erythroid 2-related factor 2; NLRP3, NLR family pyrin domain containing 3 ; g, pressure unit. 
A

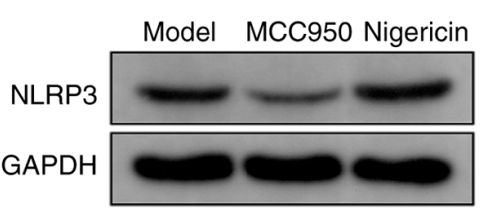

C

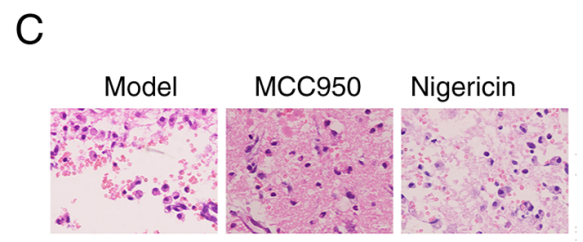

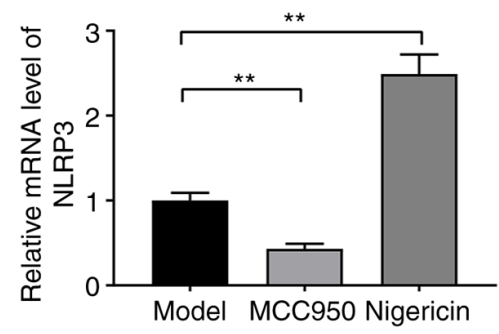
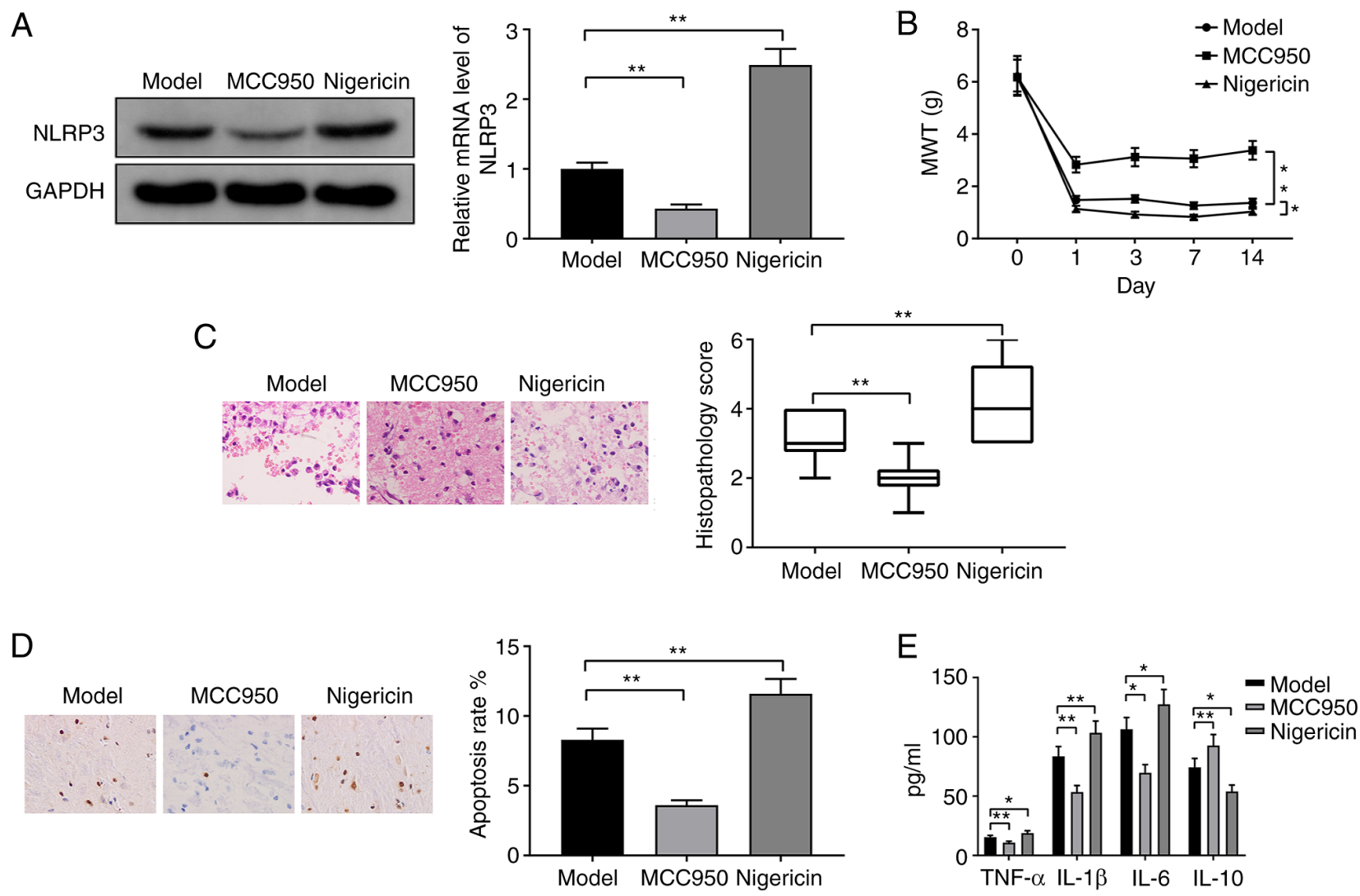

Figure 3. NLRP3 increases neuropathic pain in CCI rats. CCI rats were injected with MCC950 or Nigericin. (A) Western blotting was performed to examine the expression of NLRP3. (B) The effect of NLRP3 on the MWT of CCI rats was evaluated. (C) Hematoxylin and eosin staining revealed spinal cord injury (magnification, x400). (D) TUNEL staining was performed to examine the apoptosis in the spinal cords (magnification, $\mathrm{x} 400$ ). (E) The expression levels of inflammatory factors were measured via ELISA. $\mathrm{n}=6$ per group. ${ }^{*} \mathrm{P}<0.05 ;{ }^{* *} \mathrm{P}<0.01$. CCI, chronic constriction injury; MWT, mechanical withdrawal threshold; NLRP3, NLR family pyrin domain containing 3; g, pressure unit.

$\mathrm{P}<0.01)$. In conclusion, DEX promoted the expression of Nrf2 to alleviate neuropathic pain in CCI rats.

DEX alleviates neuropathic pain in CCI rats by suppressing $N L R P 3$ via the activation of Nrf2. To investigate the mechanism by which DEX alleviated pain, CCI rats were injected with MCC950 + ML385 or nigericin + DEX. NLRP3 was significantly upregulated in the MCC $950+$ ML385 group compared with the MCC950 group (Fig. 5A; P<0.05) and downregulated in the nigericin + DEX group compared with the nigericin group (Fig. 5A; $\mathrm{P}<0.01$ ). MWT was decreased in the MCC950 + ML385 group and increased in the nigericin + DEX group compared with the MCC950 group and nigericin group, respectively (Fig. 5B; both $\mathrm{P}<0.05$ ). Spinal cord injury and apoptosis rate were significantly increased in the MCC950 + ML385 group, but were suppressed in the nigericin + DEX group compared with the MCC950 group $(\mathrm{P}<0.05)$ and nigericin group $(\mathrm{P}<0.01)$, respectively (Fig. 5C and D). Moreover, increases in the expression levels of TNF- $\alpha$, IL- $1 \beta$ and IL- 6 and a decrease in the expression level of IL-10 were observed in the MCC950 + ML385 group compared with the MCC950 group, whereas inverse expression patterns of these cytokines were detected in the nigericin + DEX group compared with the nigericin group (Fig. 5E; all $\mathrm{P}<0.05$ ). Taken together, these results suggested that DEX inhibited NLRP3 by activating Nrf2 to suppress neuropathic pain in CCI rats.

\section{Discussion}

The diagnosis and treatment of neuropathic pain remain challenging due to the diverse etiologies and clinical manifestations of this disease (25). Over the past decades, pharmacotherapy has been recommended for the management of neuropathic pain, but only a minority of patients exhibit an adequate response (26). The present study aimed to discover the mechanism by which DEX relieved neuropathic pain, and the results suggested that DEX inhibited NLRP3 by stimulating Nrf2 to suppress neuropathic pain in a rat model of CCI.

Firstly, the neuroprotective role of DEX in a rat model of neuropathic pain was demonstrated. DEX reduced the expression levels of TNF- $\alpha$, IL- $1 \beta$ and IL- 6 while increasing the expression level of IL-10 in CCI rats. Moreover, downregulation of NLRP3 and upregulation of $\mathrm{Nrf} 2$ were also detected in the spinal cord. TNF- $\alpha$, IL-1 $\beta$ and IL- 6 have been identified as proinflammatory factors, and IL-10 has been identified as an anti-inflammatory factor in neuropathic pain (27). DEX was revealed to attenuate neuropathic pain by promoting anti-inflammatory activity in CCI (28). Consistent with the results of the current study, Farghaly et al (29) demonstrated that DEX decreased TNF- $\alpha$ and IL-6 to alleviate neuropathic pain.

In the present study, NLRP3 promoted neuropathic pain and inflammatory responses in CCI rats. Despite the lack of research on the interaction between DEX and NLRP3, the involvement of NLRP3 in neuropathic pain has been intensively investigated. 

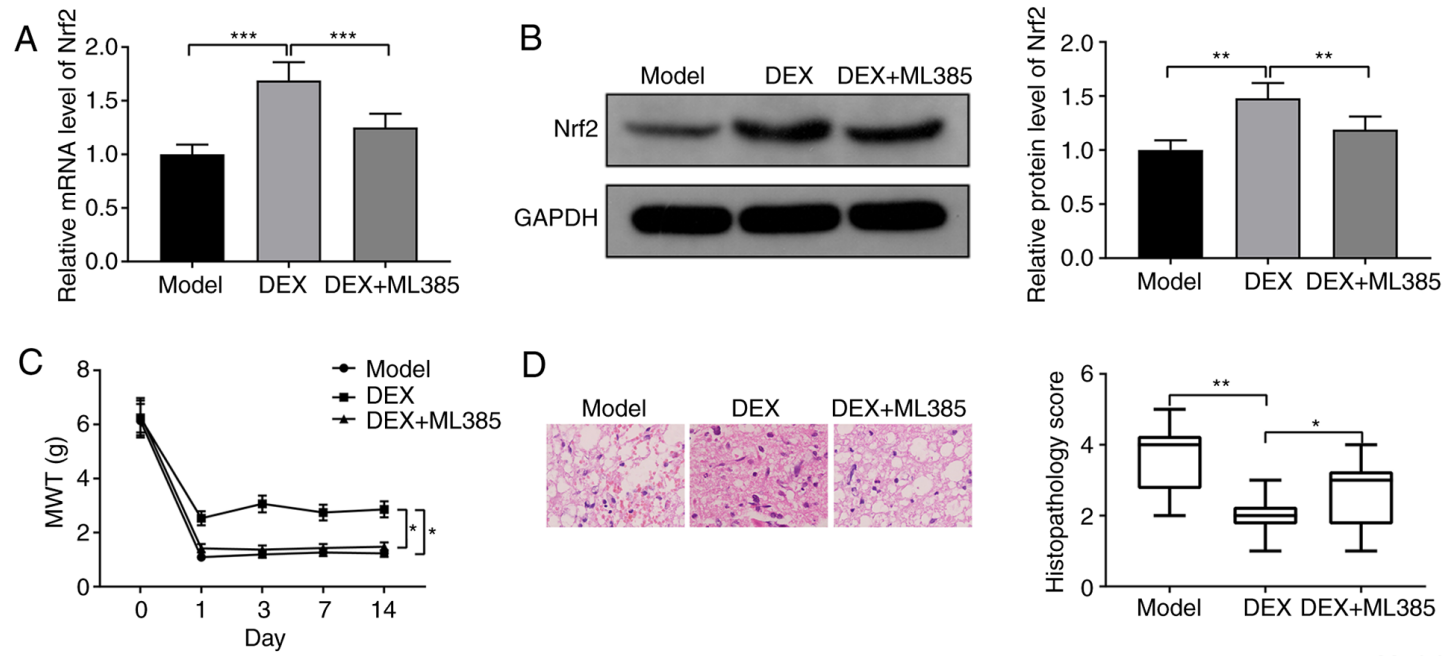

$E$
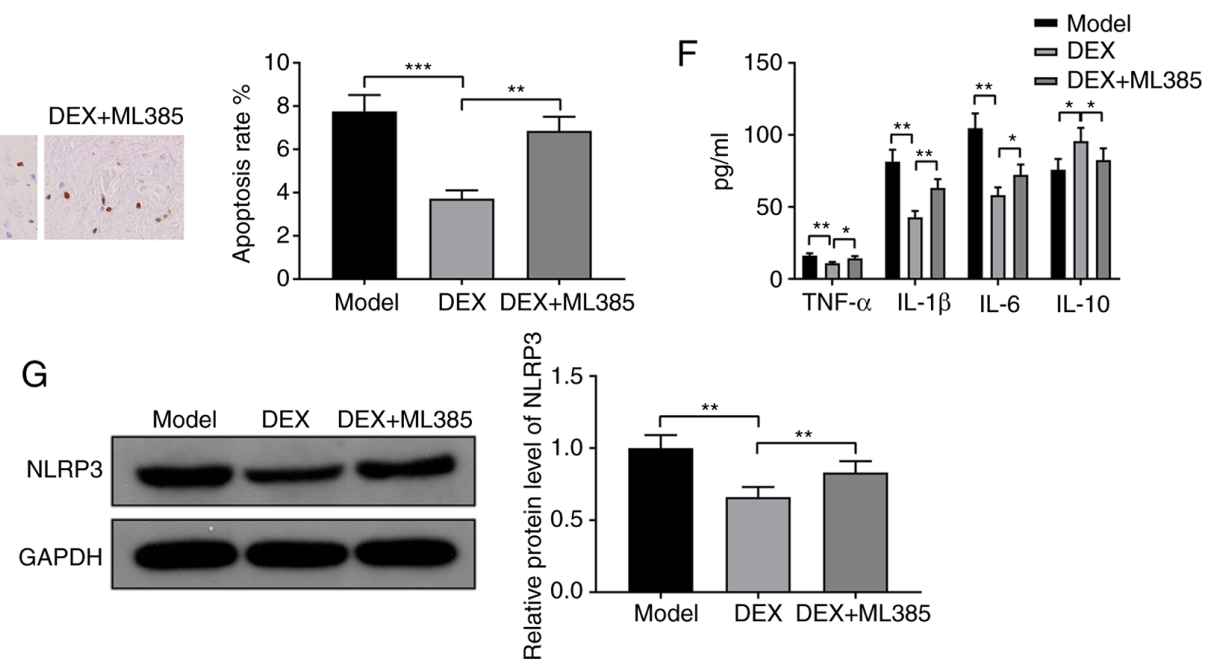

Figure 4. DEX reduces neuropathic pain by activating Nrf2 in CCI rats. CCI rats were injected with DEX and ML385. (A) Reverse transcription-quantitative PCR and (B) western blotting were performed to detect the expression of Nrf2. (C) MWT was recorded in response to mechanical stimulation. (D) Hematoxylin and eosin staining revealed spinal cord injury (magnification, $\mathrm{x} 400$ ). (E) TUNEL staining was performed to examine the apoptosis in the spinal cords (magnification, $\mathrm{x} 400$ ). (F) The expression levels of inflammatory factors were measured via ELISA. (G) Western blotting was performed to detect the expression of NLRP3. $\mathrm{n}=6$ per group. ${ }^{*} \mathrm{P}<0.05 ;{ }^{* *} \mathrm{P}<0.01 ;{ }^{* * * *} \mathrm{P}<0.001$. DEX, dexmedetomidine; CCI, chronic constriction injury; MWT, mechanical withdrawal threshold; Nrf2, nuclear factor erythroid 2-related factor 2; NLRP3, NLR family pyrin domain containing 3; g, pressure unit.

For example, microRNA-223 has been indicated to ameliorate morphine-induced analgesic tolerance to neuropathic pain by downregulating NLRP3 (30). Peptide 5 has been demonstrated to induce analgesia by inhibiting NLRP3 to protect rats from peripheral nerve injury (31). Furthermore, paclitaxel has been revealed to induce neuropathic pain by stimulating the NLRP3 inflammasome and the proinflammatory factor IL-1 $\beta$ (32).

The present study demonstrated that DEX relieved neuropathic pain via the activation of Nrf2. CCI rats that were treated with DEX and ML385 exhibited exacerbated neuropathic pain and upregulated NLRP3 expression. A previous study showed that the expression of $\mathrm{Nrf} 2$ was reduced in a rat model of chronic neuropathic pain (33). Nrf2 may alleviate oxaliplatin-induced peripheral neuropathy by maintaining mitochondrial homeostasis and suppressing oxidative stress (34). Moreover, rutin protects against diabetic neuropathy by inhibiting plasma glucose levels and neuroinflammation through the activation of Nrf2 signaling (35). However, to the best of our knowledge, the regulation of Nrf2 in DEX-induced neuroprotection is largely unknown.

The NLRP3/Nrf2 pathway has been extensively investigated in numerous pharmacological and pathological processes. For example, mangiferin has been indicated to reduce lipopolysaccharide- and D-galactosamine-induced liver injury by inhibiting inflammation and oxidative stress via suppression of NLRP3 and activation of Nrf2 (36). Dimethyl fumarate has been revealed to alleviate dextran sulfate sodium-induced colitis by stimulating Nrf2-mediated suppression of the NLRP3 inflammasome (37). Sulforaphane has been demonstrated to protect acinar cells from acute pancreatitis by regulating Nrf2-mediated oxidative stress and the NLRP3 inflammasome (38). In the present study, NLRP3 was upregulated in MCC950-treated CCI rats in response to ML385 injection, indicating the suppressive effect of Nrf2 on NLRP3 expression. Furthermore, DEX was indicated to downregulate NLRP3 in nigericin-treated CCI rats, resulting in a reduction in the MWT, as well as spinal cord injury and inflammatory responses. Based on the aforementioned protective effect of $\mathrm{Nrf2}$, it was concluded that DEX relieved neuropathic pain in CCI rats by suppressing NLRP3 via the activation of Nrf2. Additionally, Nrf2 has been revealed to modulate HO-1 in dietary oleuropein- and peracetylated oleuropein-mediated suppression of murine 


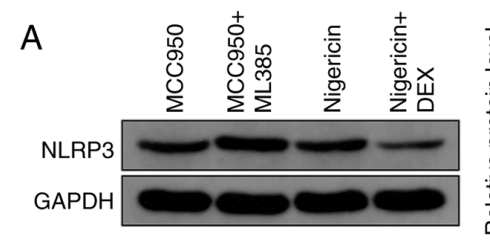

C

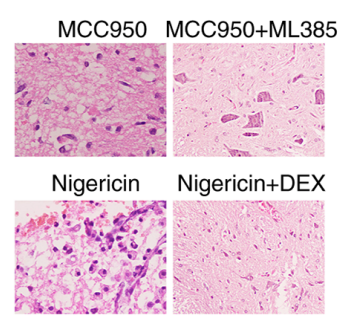

D

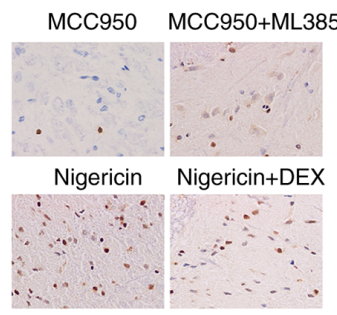

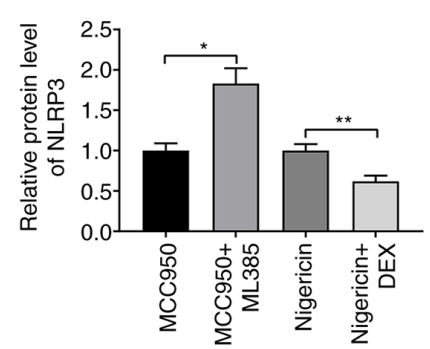

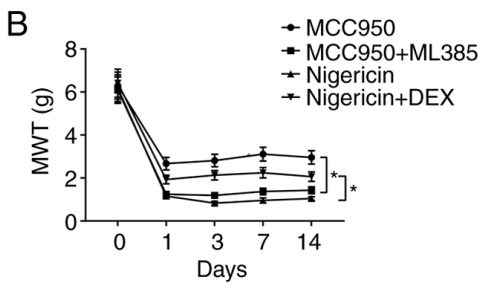

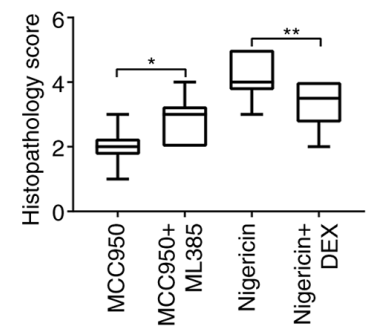

E

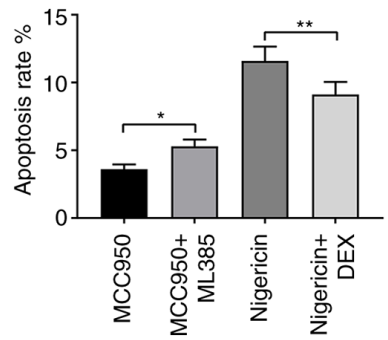

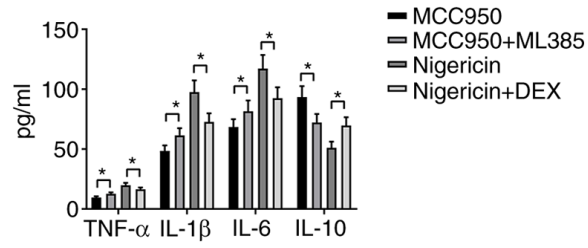

Figure 5. DEX relieves neuropathic pain in CCI rats by suppressing NLRP3 via activation of Nrf2. CCI rats were injected with MCC950 + ML385 or Nigericin + DEX (A) Western blotting was performed to detect the expression of NLRP3. (B) MWT was recorded in response to mechanical stimulation. (C) Hematoxylin and eosin staining revealed spinal cord injury (magnification, $\mathrm{x} 400$ ). (D) TUNEL staining was performed to examine the apoptosis in the spinal cords (magnification, $\mathrm{x} 400$ ). (E) The expression levels of inflammatory factors were measured via ELISA. $n=6$ per group. ${ }^{*} \mathrm{P}<0.05 ;{ }^{* *} \mathrm{P}<0.01$. DEX, dexmedetomidine; $\mathrm{CCI}$, chronic constriction injury; MWT, mechanical withdrawal threshold; Nrf2, nuclear factor erythroid 2-related factor 2; NLRP3, NLR family pyrin domain containing 3; g, pressure unit.

lupus nephritis (39). Further studies should be performed to explore whether a downstream target of Nrf2 is involved in the management of neuropathic pain.

The present study had several limitations that should be mentioned. Firstly, only the mechanism underlying the effect of DEX in animal models was examined, and this mechanism should be further clarified in vitro and clinically. In the present study, DEX relieved neuropathic pain by activating Nrf2 to inhibit NLRP3-mediated neuroinflammation. Targeting the Nrf2/NLRP3 signaling pathway may be a novel intervention method for neuropathic pain treatment. However, it has been demonstrated that DEX exerts neuroprotective and analgesic effects via the microRNA-101/E2F2/toll-like receptor $4 / \mathrm{NF}-\kappa \mathrm{B}$ axis (40). Whether this signaling pathway is targeted in addition to the Nrf2/NLRP3 signaling pathway in the context of DEX treatment requires further elucidation. Additionally, the analgesic effect of DEX should be clinically evaluated through preliminary clinical pharmacological and safety analyses and pharmacokinetic tests.

In summary, DEX ameliorated neuropathic pain in a rat model of CCI. The NLRP3/Nrf2 pathway was verified to modulate inflammatory responses in neuropathic pain. DEX inhibited neuropathic pain by activating Nrf2 through the suppression of NLRP3. Understanding the mechanism of DEX may contribute to the alleviation of neuropathic pain in patients and improve the pharmacotherapeutic treatment of neuropathy.

\section{Acknowledgements}

Not applicable.

\section{Funding}

No funding was received.

\section{Availability of data and materials}

The datasets used and/or analyzed during the current study are available from the corresponding author on reasonable request.

\section{Authors' contributions}

WS and JL conceived the study design. WS and JL designed the experiments. XL performed the experiments. YT analyzed the data. JL and WS provided critical materials. WS and XL wrote the manuscript. JL supervised the study. WS and JL confirm the authenticity of all the raw data. All authors have read and approved the final manuscript.

\section{Ethics approval and consent to participate}

All animal experiments were approved by the Animal Protection and Use Committee of Hunan Provincial People's Hospital (Changsha, China) and strictly followed 
the 'Guidelines for the Use and Management of Laboratory Animals' issued by the National Institutes of Health and Animal Research: Reporting In Vivo Experiments Guidelines.

\section{Patient consent for publication}

Not applicable.

\section{Competing interests}

The authors declare that they have no competing interests.

\section{References}

1. Bouhassira D and Attal N: Translational neuropathic pain research: A clinical perspective. Neuroscience 338: 27-35, 2016.

2. Gierthmuhlen J and Baron R: Neuropathic pain. Semin Neurol 36: 462-468, 2016.

3. Scholz J, Finnerup NB, Attal N, Aziz Q, Baron R, Bennett MI, Benoliel R, Cohen M, Cruccu G, Davis KD, et al: The IASP classification of chronic pain for ICD-11: Chronic neuropathic pain. Pain 160: 53-59, 2019

4. Colloca L, Ludman T, Bouhassira D, Baron R, Dickenson AH, Yarnitsky D, Freeman R, Truini A, Attal N, Finnerup NB, et al: Neuropathic pain. Nat Rev Dis Primers 3: 17002, 2017.

5. Gilron I, Baron R and Jensen T: Neuropathic pain: Principles of diagnosis and treatment. Mayo Clin Proc 90: 532-545, 2015.

6. Weerink MAS, Struys MMRF, Hannivoort LN, Barends CRM, Absalom AR and Colin P: Clinical pharmacokinetics and pharmacodynamics of dexmedetomidine. Clin Pharmacokinet 56: 893-913, 2017.

7. Keating GM: Dexmedetomidine: A review of its use for sedation in the intensive care setting. Drugs 75: 1119-1130, 2015

8. Jessen Lundorf L, Korvenius Nedergaard H and Moller AM Perioperative dexmedetomidine for acute pain after abdominal surgery in adults. Cochrane Database Syst Rev 2: CD010358, 2016

9. Lin JP, Chen CQ, Huang LE, Li NN, Yang Y, Zhu SM and Yao YX: Dexmedetomidine attenuates neuropathic pain by inhibiting P2X7R expression and ERK phosphorylation in rats. Exp Neurobiol 27: 267-276, 2018.

10. Zhao Y, He J, Yu N, Jia C and Wang S: Mechanisms of dexmedetomidine in neuropathic pain. Front Neurosci 14: 330, 2020.

11. Jo EK, Kim JK, Shin DM and Sasakawa C: Molecular mechanisms regulating NLRP3 inflammasome activation. Cell Mol Immunol 13: 148-159, 2016.

12. He Y, Hara H and Nunez G: Mechanism and regulation of NLRP3 inflammasome activation. Trends Biochem Sci 41: 1012-1021, 2016.

13. Coll RC, Robertson AA, Chae JJ, Higgins SC, Munoz-Planillo R, Inserra MC, Vetter I, Dungan LS, Monks BG, Stutz A, et al: A small-molecule inhibitor of the NLRP3 inflammasome for the treatment of inflammatory diseases. Nat Med 21: 248-255, 2015.

14. Grace PM, Strand KA, Galer EL, Urban DJ, Wang X, Baratta MV, Fabisiak TJ, Anderson ND, Cheng K, Greene LI, et al: Morphine paradoxically prolongs neuropathic pain in rats by amplifying spinal NLRP3 inflammasome activation. Proc Natl Acad Sci USA 113: E3441-E3450, 2016.

15. Ming T, Yuan M, Kong Q, Huang Q, Xia Z and Wu X: Dexmedetomidine alleviates blunt chest trauma and hemorrhagic shock-resuscitation-induced acute lung injury through inhibiting the NLRP3 inflammasome. Mol Med Rep 22: 2507-2515, 2020.

16. Yang T, Feng X, Zhao Y,Zhang H, Cui H, Wei M, Yang H and Fan H: Dexmedetomidine enhances autophagy via $\alpha 2$-AR/AMPK/mTOR pathway to inhibit the activation of NLRP3 inflammasome and subsequently alleviates lipopolysaccharide-induced acute kidney injury. Front Pharmacol 11: 790, 2020.

17. Ahmed SM, Luo L, Namani A, Wang XJ and Tang X: Nrf2 signaling pathway: Pivotal roles in inflammation. Biochim Biophys Acta Mol Basis Dis 1863: 585-597, 2017.

18. Thimmulappa RK, Lee H, Rangasamy T, Reddy SP Yamamoto M, Kensler TW and Biswal S: Nrf2 is a critical regulator of the innate immune response and survival during experimental sepsis. J Clin Invest 116: 984-995, 2006.

19. Arruri V, Komirishetty P, Areti A, Dungavath SK and Kumar A: Nrf2 and NF- $\kappa$ B modulation by Plumbagin attenuates functional, behavioural and biochemical deficits in rat model of neuropathic pain. Pharmacol Rep 69: 625-632, 2017.
20. Du L, Wang J, Chen Y, Li X, Wang L, Li Y, Jin X, Gu X, Hao M, Zhu X, et al: Novel biphenyl diester derivative AB-38b inhibits NLRP3 inflammasome through Nrf2 activation in diabetic nephropathy. Cell Biol Toxicol 36: 243-260, 2020.

21. Li F, Wang X, Zhang Z, Zhang X and Gao P: Dexmedetomidine attenuates neuroinflammatory-induced apoptosis after traumatic brain injury via Nrf2 signaling pathway. Ann Clin Transl Neurol 6: 1825-1835, 2019.

22. Kilkenny C, Browne W, Cuthill IC, Emerson M and Altman DG; NC3Rs Reporting Guidelines Working Group: Animal research: Reporting in vivo experiments: The ARRIVE guidelines. J Gene Med 12: 561-563, 2010.

23. Wiesenfeld-Hallin Z: Sex differences in pain perception. Gend Med 2: 137-145, 2005

24. Livak KJ and Schmittgen TD: Analysis of relative gene expression data using real-time quantitative PCR and the 2 (-Delta Delta C(T)) Method. Methods 25: 402-408, 2001.

25. Cruccu $G$ and Truini A: A review of neuropathic pain: From guidelines to clinical practice. Pain Ther 6 (Suppl 1): S35-S42, 2017.

26. Attal N and Bouhassira D: Pharmacotherapy of neuropathic pain: Which drugs, which treatment algorithms? Pain 156 (Suppl 1): S104-S114, 2015.

27. Lees JG, Fivelman B, Duffy SS, Makker PG, Perera CJ and Moalem-Taylor G: Cytokines in neuropathic pain and associated depression. Mod Trends Pharmacopsychiatry 30: 51-66, 2015.

28. Liang F, Liu M, Fu X, Zhou X, Chen $P$ and Han F: Dexmedetomidine attenuates neuropathic pain in chronic constriction injury by suppressing NR2B, NF- $\kappa \mathrm{B}$, and iNOS activation. Saudi Pharm J 25: 649-654, 2017.

29. Farghaly HS, Mahmoud AM and Abdel-Sater KA: Effect of dexmedetomidine and cold stress in a rat model of neuropathic pain: Role of interleukin- 6 and tumor necrosis factor- $\alpha$. Eur J Pharmacol 776: 139-145, 2016.

30. Xie XJ, Ma LG, Xi K, Fan DM, Li JG, Zhang Q and Zhang W: Effects of microRNA-223 on morphine analgesic tolerance by targeting NLRP3 in a rat model of neuropathic pain. Mol Pain 13: 1744806917706582, 2017.

31. Tonkin RS, Bowles C, Perera CJ, Keating BA, Makker PG, Duffy SS, Lees JG, Tran C, Don AS, Fath T, et al: Attenuation of mechanical pain hypersensitivity by treatment with Peptide5, a connexin-43 mimetic peptide, involves inhibition of NLRP3 inflammasome in nerve-injured mice. Exp Neurol 300: 1-12, 2018.

32. Jia M, Wu C, Gao F, Xiang H, Sun N, Peng P, Li J, Yuan X, Li H, Meng X, et al: Activation of NLRP3 inflammasome in peripheral nerve contributes to paclitaxel-induced neuropathic pain. Mol Pain 13: 1744806917719804, 2017

33. Li S, Yang C, Fang X, Zhan G, Huang N, Gao J, Xu H, Hashimoto $\mathrm{K}$ and Luo A: Role of Keap1-Nrf2 signaling in anhedonia symptoms in a rat model of chronic neuropathic pain: Improvement with sulforaphane. Front Pharmacol 9: 887, 2018.

34. Yang Y, Luo L, Cai X, Fang Y, Wang J, Chen G, Yang J, Zhou Q, Sun X, Cheng X, et al: Nrf2 inhibits oxaliplatin-induced peripheral neuropathy via protection of mitochondrial function. Free Radic Biol Med 120: 13-24, 2018.

35. Tian R, Yang W, Xue Q, Gao L, Huo J, Ren D and Chen X: Rutin ameliorates diabetic neuropathy by lowering plasma glucose and decreasing oxidative stress via Nrf2 signaling pathway in rats. Eur J Pharmacol 771: 84-92, 2016.

36. Pan CW, Pan ZZ, Hu JJ, Chen WL, Zhou GY, Lin W, Jin LX and $\mathrm{Xu} C \mathrm{CL}$ : Mangiferin alleviates lipopolysaccharide and D-galactosamine-induced acute liver injury by activating the Nrf2 pathway and inhibiting NLRP3 inflammasome activation. Eur J Pharmacol 770: 85-91, 2016.

37. Liu X, Zhou W, Zhang X, Lu P, Du Q, Tao L, Ding Y, Wang Y and Hu R: Dimethyl fumarate ameliorates dextran sulfate sodium-induced murine experimental colitis by activating Nrf2 and suppressing NLRP3 inflammasome activation. Biochem Pharmacol 112: 37-49, 2016.

38. Dong Z, Shang H, Chen YQ, Pan LL, Bhatia M and Sun J: Sulforaphane protects pancreatic acinar cell injury by modulating Nrf2-Mediated oxidative stress and NLRP3 inflammatory pathway. Oxid Med Cell Longev 2016: 7864150, 2016.

39. Castejon ML, Sanchez-Hidalgo M, Aparicio-Soto M Montoya T, Martin-LaCave I, Fernandez-Bolanos JG and Alarcon-de-la-Lastra C: Dietary oleuropein and its new acyl-derivate attenuate murine lupus nephritis through $\mathrm{HO}-1 / \mathrm{Nrf} 2$ activation and suppressing JAK/STAT, NF- $\mathrm{B}$, MAPK and NLRP3 inflammasome signaling pathways. J Nutr Biochem 74: 108229, 2019

40. Zhang W, Yu T, Cui X, Yu H and Li X: Analgesic effect of dexmedetomidine in rats after chronic constriction injury by mediating microRNA-101 expression and the E2F2-TLR4-NF-kB axis. Exp Physiol 105: 1588-1597, 2020.

(i) $(9)$ This work is licensed under a Creative Commons Attribution-NonCommercial-NoDerivatives 4.0 International (CC BY-NC-ND 4.0) License. 